\title{
Resultados de la plastia de oposición de Camitz en pacientes con atrofia tenar secundaria a síndrome de canal del carpo grave crónico
}

\author{
F. Lajara Marco ${ }^{(1)}$, A. Fuentes Díaz ${ }^{(2)}$, P. Martínez Sáez ${ }^{(3)}$, R. Lax Pérez ${ }^{(4)}$ \\ (1) Mir DE C.O.T. Hospital VeGa BAJA. ORIhuela (ALICANTE) \\ (2) JEFE DE SECCIÓN SERVICIO C.O.T. HoSPITAL VEGA BAJA. ORIHUELA (ALICANTE) \\ (3) F.E.A. SERVICIO DE C.O.T. Hospital REINA SofÍa (MURCIa) \\ (4) F.E.A. SERVICIO DE C.O.T. Hospital VeGa BAJA. ORIHUELa (AliCANTE)
}

Correspondencia:

Dr. Francisco Lajara Marco

Ctra. Orihuela-Almoradí, s/n

03314 San Bartolomé (Orihuela). Alicante

Teléf: 965877500

e-mail: pacolajara@hotmail.com

Objetivo. Evaluar los resultados funcionales de la trasferencia del palmar menor en los casos con atrofia tenar secundaria a síndrome del canal carpiano crónico.

Material y Método. Se revisan retrospectivamente 6 pacientes con una media de edad de 63 años e igual distribución en cuanto a género. En cuatro casos se trató de la mano dominante. Se registraron: el grado de atrofia, la movilidad del pulgar, y la fuerza. Se calcularon el DASH y el CTS y se registraron las complicaciones.

Resultados. Tras 45,7 meses de seguimiento medio, la abducción radial fue de $53,3^{\circ}$ y todos lograron la oposición completa. Las pinzas pulpejo-pulpejo y pulpejo-lateral y la fuerza de puño fueron respectivamente del $75,1 \%, 96,6 \%$ y $96,8 \%$ respecto al lado contralateral. Se registró una mejoría significativa en los cuestionarios DASH y CTS siendo los valores del ES y SRM mayores de 0,8 en todos los casos.

Conclusiones. Creemos que la plastia de oposición de Camitz es una intervención simple, segura y efectiva que ayuda a mejorar la función de la mano en
Aim: The purpose of this study was to evaluate the results of transfer of the palmaris longus in thenar paralysis caused by advanced chronic carpal tunnel syndrome.

Methods: This is a retrospective review of six patients with an average of age of 63 years, 3 men and 3 women. We analyzed thenar muscle recovery, thumb mobility, power and functional grading using DASH and CTS questionnaires as well as complications encountered.

Results: The mean duration of follow-up was 45,7 months, the mean of radial abduction was $53,3^{\circ}$, the range of opposition was complete. The tip and keypinch and grip power were $75,1 \%, 96,6 \%$ y $96,8 \%$ respectively according to the contralateral values. There was significant improvement in DASH and CTS score with values of ES and SRM over 0,8 in all patients.

Conclusions: The Camitz operation is a simple, effective, and safe means of improving hand function in elderly patients with long-standing carpal tunnel syndrome and thenar muscle atrophy. 
los casos de SCC crónicos graves, especialmente en pacientes mayores.

Palabras clave: síndrome del canal carpiano, atrofia tenar, plastia de oposición.
Key words: carpal tunnel syndrome, thenar muscle atrophy, opponensplasty.

Rev. Iberam. Cir. Mano - Vol. 37 • Núm. 1 Mayo 2009 (30-37)

\section{INTRODUCCIÓN}

$\mathrm{P}$ ara la realización de las actividades de la vida diaria que requieren de una pinza fina y para la destreza de la mano en general, se precisa en gran medida de la oposición del pulgar. La atrofia tenar es habitual en pacientes con síndrome del canal carpiano (SCC) de larga evolución ${ }^{1,2}$, desafortunadamente, su recuperación tras la liberación del nervio mediano es bastante infrecuente, especialmente si se trata de pacientes mayores ${ }^{3,4}$. Se han descrito numerosos procedimientos destinados a mejorar la oposición del pulgar ${ }^{5-7}$, aunque el usado más frecuentemente es la plastia con palmaris longus descrita por primera vez por $\mathrm{Camitz}^{8}$. El objetivo de este estudio es evaluar los resultados funcionales de la trasposición del palmaris longus para restaurar la oposición del pulgar en los casos con atrofia tenar grave secundaria a SCC crónico.

\section{MATERIAL Y MÉTODO}

Entre febrero de 2002 y diciembre de 2008 fueron operados en el Hospital Vega Baja de
Orihuela (Alicante), por un mismo cirujano, 154 pacientes (224 casos) con SCC. Todos ellos presentaban una sintomatología de al menos un año de evolución y confirmación electromiográfica (EMG) de compresión nerviosa. Las reintervenciones y los pacientes en los que el cuadro podría estar relacionado con procesos generales como diabetes, hipotiroidismo o artritis reumatoidea fueron excluidos. Finalmente, seis casos $(2,67 \%)$ fueron seleccionados para estudio, por ser: SCC crónicos con debilidad en la abducción del pulgar y atrofia tenar en los que a la liberación del ligamento anular del carpo se asoció una trasposición del palmaris longus mediante la técnica descrita por $\mathrm{Camitz}^{8}$.

$\mathrm{Su}$ media de edad era de 63,7 años (rango 52-76 años), con igual distribución en cuanto al género. En cuatro casos la afectada fue la mano derecha, que era la dominante (Tabla I). El diagnóstico de SCC se estableció apoyándose en los síntomas y signos (dolor nocturno o por el día, hormigueos y parestesias, debilidad y atrofia de la musculatura tenar, disminución de la discriminación de dos puntos, Tinel y Phalen positivos), y en la EMG que informaba de compresión grave del nervio ${ }^{10}$. La atrofia tenar se clasificó según

Tabla I - CARACterísticas dE NUESTRA SERIE

\begin{tabular}{|c|c|c|c|c|c|c|}
\hline Casos & Género & Edad & $\begin{array}{l}\text { Lado afecto } \\
\text { (Dominante) }\end{array}$ & $\begin{array}{l}\text { Seguimiento } \\
\text { (meses) }\end{array}$ & Profesión & $\begin{array}{c}\text { Reinserción } \\
\text { laboral }\end{array}$ \\
\hline 1 & $M$ & 52 & (D) & 45,6 & Frutera & Sí \\
\hline 2 & $\mathrm{M}$ & 70 & 1 & 70 & Ex Carnicera & - \\
\hline 3 & $\mathrm{H}$ & 76 & (D) & 4 & Ex Electricista & - \\
\hline 4 & $\mathrm{H}$ & 67 & (D) & 13 & Ex Cantero & - \\
\hline 5 & $\mathrm{H}$ & 64 & I & 83,7 & Ex Albañil & - \\
\hline 6 & $M$ & 53 & (D) & 70,3 & Cocinera & Sí \\
\hline
\end{tabular}

H: hombre; M: mujer; D: derecha; I: izquierda. 


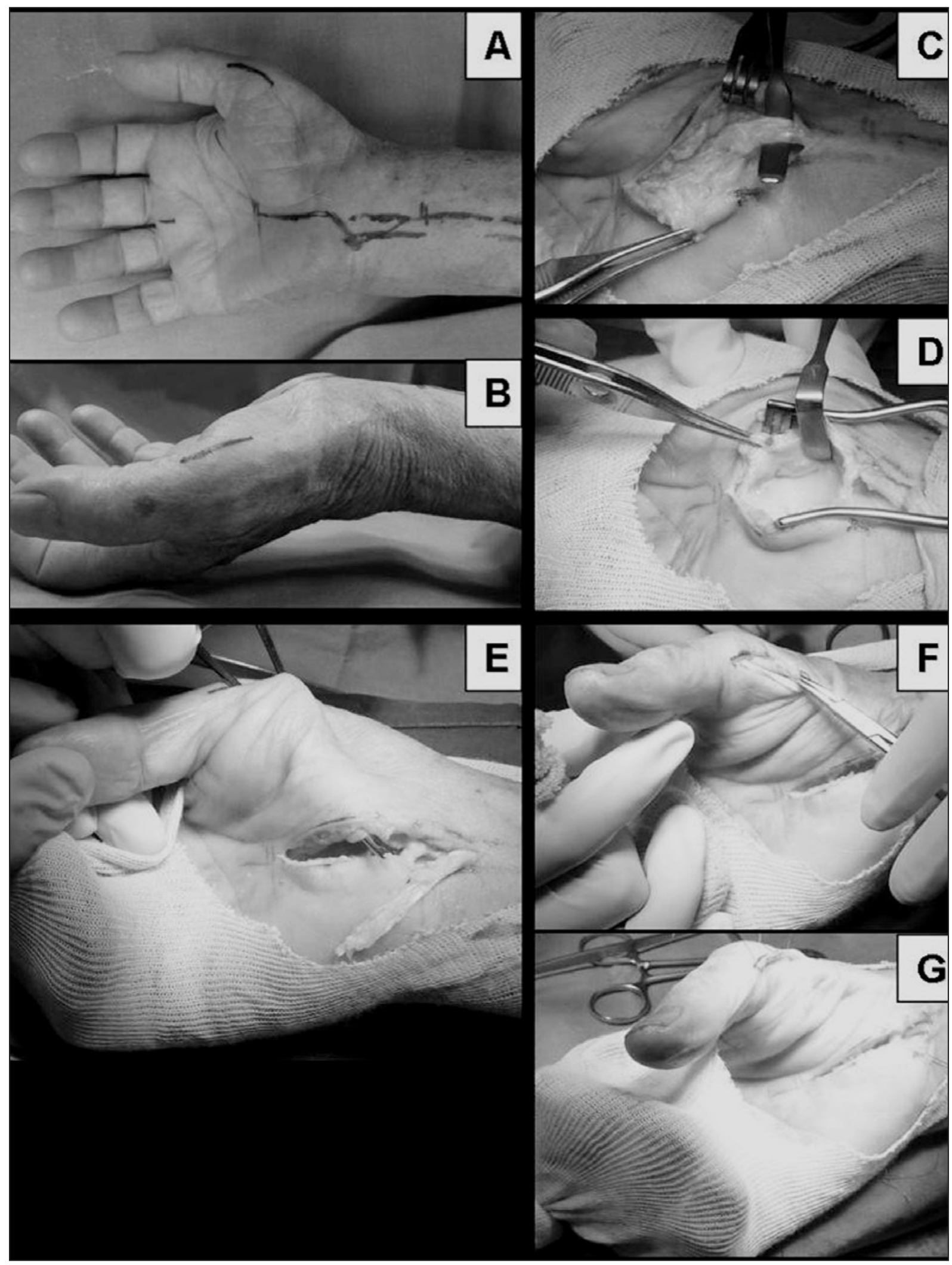

Figura 1. Técnica quirúrgica de la intervención correspondiente al caso 3. A. Incisión quirúrgica. B. Atrofia tenar grave. C. Disección del palmaris longus con una prolongación de la aponeurosis palmar. D. Liberación del nervio mediano en el canal carpiano. E. Tunelización subcutánea de las 2 incisiones. F. Sutura de la plastia a la inserción del abductor pollicis brevis. G. Ajuste de la tensión de la plastia. 
Gelberman et al. ${ }^{9}$ encontrando 5 casos graves y uno moderado. Como factores de co-morbilidad: hubo un caso asociaba un pulgar en resorte y una enfermedad de De Quervain ipsilateral que se trataron en el mismo acto quirúrgico (caso $\mathrm{n}^{\circ} 1$ ). Y otros 2 casos padecían SCC contralateral que fue intervenido 4 meses después mediante descompresión simple (casos $\mathrm{n}^{\mathrm{o}} 2$ y n $^{\mathrm{o}} 4$ ).

Técnica quirúrgica: Bajo anestesia regional intravenosa o bloqueo troncular nervioso de los nervios mediano-cubital y ramas sensitivas del radial a nivel de la muñeca, se realiza una incisión de unos $2 \mathrm{~cm}$ de longitud en el eje palmar del dedo anular proximal a la línea de Kaplan, a nivel del pliegue de flexión de la muñeca se curva oblicuamente extendiéndose proximalmente en el antebrazo volar otros $2 \mathrm{~cm}$ (Figura 1A). La rama cutánea del nervio mediano debe ser identificada y preservada cerca de la inserción del palmaris longus en la aponeurosis palmar. Éste es disecado distalmente con una prolongación de unos 2 a $4 \mathrm{~cm}$ de dicha aponeurosis (Figura 1C). Una vez disecado el tendón, se abre el ligamento anular del carpo, cuidando de no lesionar la rama motora del nervio mediano (Figura 1D). Se realiza una segunda incisión de $1 \mathrm{~cm}$ de longitud en el lado radial del pulgar a nivel de la articulación metacarpofalángica y creamos un túnel subcutáneo que una ambas incisiones (Figura 1E). Pasamos a su través el palmaris longus junto con la prolongación de la aponeurosis y los suturamos a la inserción del abductor pollicis brevis (también se puede suturar a la cápsula dorsal ó al extensor pollicis brevis) (Figura 1F). La tensión de la plastia es ajustada de forma que, en extensión y en flexión pasiva de muñeca, se consiga respectivamente la máxima oposición y extensión del pulgar (Figura 1G). Posteriormente se coloca una férula durante 4 semanas con el pulgar y la muñeca en abducción y extensión media. Tras la retirada de la inmovilización se permitió la movilización libre de la muñeca y dedos ${ }^{11}$.

Para el análisis de los resultados principales, extraídos de la versión española de los cuestionarios DASH (Disabilities of the Arm, Shoulder, and Hand) y CTS (Carpal Tunnel Syndrome) ${ }^{12-14}$ se emplea un diseño clínico tipo cohortes histórico basado en mediciones antes y después de la cirugía de forma retrospectiva. Al final de seguimiento, se calculó la mejoría en la calidad de vida de los pacientes utilizando los cuestionarios DASH y CTS determinando su respuesta en función al «tamaño del efecto» (ES) (media de la diferencia pre-postratamiento/ desviación estándar de la línea base) y en relación también a la Media Estandarizada de la respuesta o SRM (media de la diferencia/ desviación estándar de la diferencia) ${ }^{15}$, definiendo el cambio como pequeño cuando era $\leq 0,2$, medio $\leq 0,5$ y grande $\geq 0,8$.

Sin embargo, para los resultados secundarios, basados en medidas durante examen clínico realizadas por un examinador independiente, se ha usado un diseño descriptivo de corte transverso: con una sola medición en el tiempo al final de seguimiento en el caso de la movilidad del pulgar (abducción radial, la abducción palmar máxima ${ }^{16}$ y la oposición mediante el índice de Kapandji ${ }^{17}$ ), y comparando la medición con la mano contralateral y con medidas en población general realizadas por otros autores en estudios previos ${ }^{18,19}$, denominadas «teóricas» según edad, género, lado afecto y dominancia; en el caso de la fuerza de las pinzas pulpejo-pulpejo y pulpejo-lateral así como la de agarre (puño), que fueron medidas mediante pinzómetro y dinamómetro de JAMAR ${ }^{20}$.

\section{RESULTADOS}

Como complicaciones sólo encontramos un caso de disestesia transitoria de la rama sensitiva del nervio mediano en el recorrido de la plastia a nivel de la eminencia tenar. No tuvimos ningún caso de hipersensibilidad de la cicatriz quirúrgica, dolor cicatricial («pillar-pain»), lesiones del nervio mediano, infección ni de síndrome doloroso regional complejo.

Tras un seguimiento medio de 45,7 meses (rango 4-83,7 meses), el DASH mejoró una media de 54,1 puntos (rango 30-71,6), con un ES de 6 y una SRM de 3,45; el cuestionario CTS para la valoración funcional descendió con la intervención de 3,16 puntos preoperatorios a 1,16 puntos al final del período de seguimiento con un ES de 2,25 y una SRM de 2,51; con respecto a la gravedad de síntomas también descendió desde 3,89 puntos a 1,27 puntos de media, con un ES de 11,25 y una SRM de 6,12. 


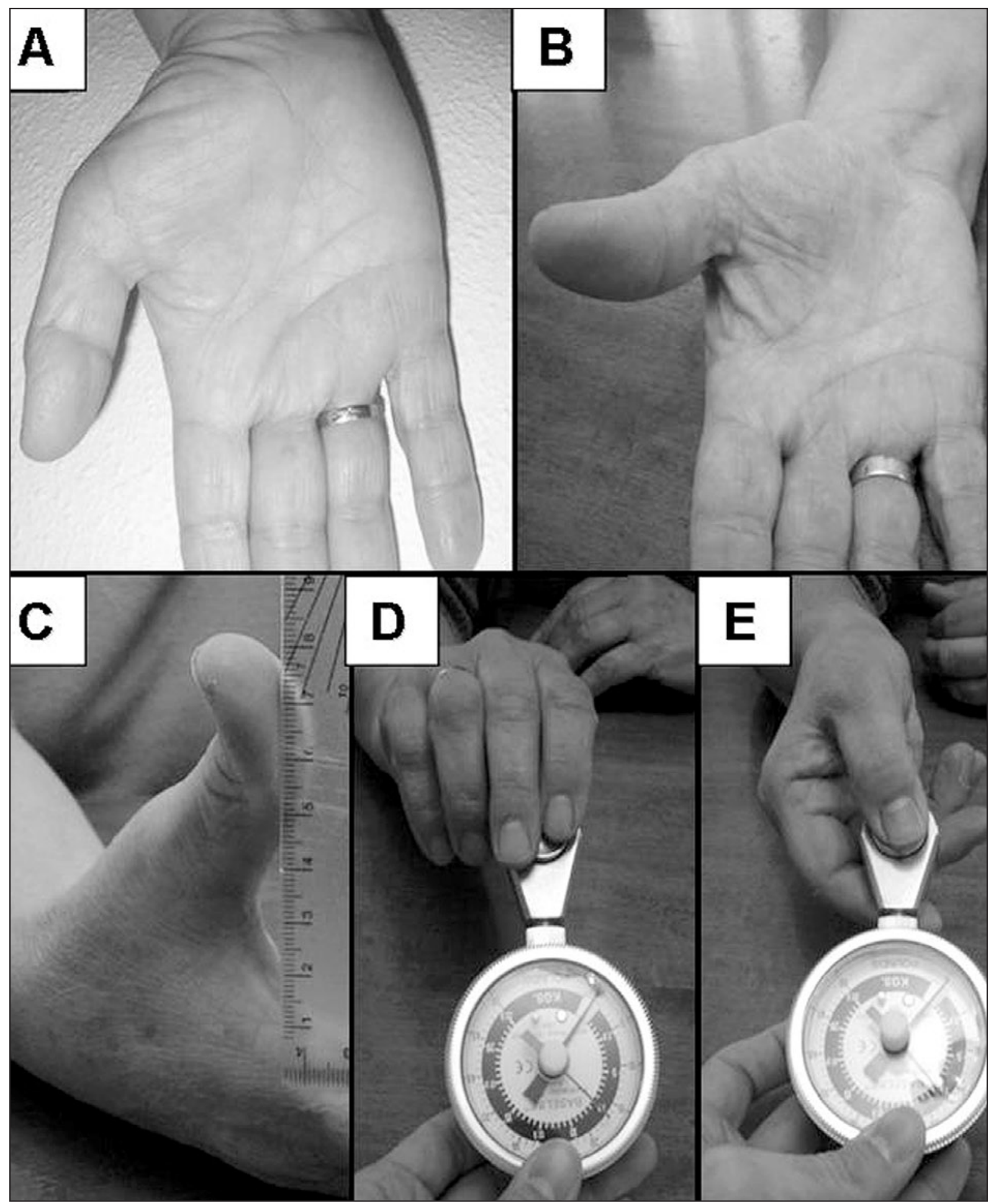

Figura 2. Caso $n^{o}$ 1. A. Atrofia tenar moderada. B. Aspecto a los 45 meses donde se observa una recuperación parcial de la atrofia tenar con una buena función. C. Abducción palmar máxima de $8 \mathrm{~cm}$. D. Pinza pulpejo-lateral 8 Kg. E. Pinza pulpejo-pulpejo de $7,5 \mathrm{Kg}$.

En cuanto a la reinserción laboral, los 2 pacientes que trabajaban volvieron a su ocupación previa (Tabla II).
Respecto a la atrofia muscular, sólo se observó mejoría en uno de los seis pacientes, pasando a ser ésta de moderada a ligera (Figura 2). En 


\section{Tabla II - RESULTADOS DE LA VALORACIÓN SUBJETIVA (DASH y CTS) Y REINSERCIÓN LABORAL}

\begin{tabular}{|c|c|c|c|c|c|c|c|}
\hline Caso & $\begin{array}{c}\text { DASH preQx/ } \\
\text { postQx }\end{array}$ & $\begin{array}{c}\text { Mejoría } \\
\text { DASH }\end{array}$ & $\begin{array}{c}\text { CTS RF pre- } \\
\text { Qx/postQx }\end{array}$ & $\begin{array}{c}\text { Mejoría } \\
\text { CTS RF }\end{array}$ & $\begin{array}{c}\text { CTS GS pre- } \\
\text { Qx/postQx }\end{array}$ & $\begin{array}{c}\text { Mejoría } \\
\text { CTS SS }\end{array}$ & $\begin{array}{c}\text { Reinserción } \\
\text { laboral }\end{array}$ \\
\hline 1 & $71,6 / 0$ & 71,6 & $3,1 / 1$ & 2,1 & $4 / 1$ & 3 & Sí \\
2 & $80 / 50$ & 30 & $4,1 / 1,2$ & 2,8 & $4,1 / 1,2$ & 2,7 & - \\
3 & $63,3 / 10,8$ & 52,5 & $3,5 / 1,2$ & 2,25 & $3,9 / 1,4$ & 2,4 & - \\
4 & $53,3 / 0$ & 53,3 & $1,5 / 1$ & 0,5 & $3,9 / 1$ & 3 & - \\
5 & $65,8 / 19$ & 46,6 & $3,5 / 1,5$ & 2 & $3,7 / 1,5$ & 1,9 & - \\
6 & $70,8 / 0$ & 70,8 & $3,25 / 1$ & 2,25 & $3,5 / 1$ & 2,5 & Sí \\
\hline Media & $67,5 / 13,3$ & 54,1 & $3,16 / 1,16$ & 2 & $3,89 / 1,27$ & 2,62 & \\
DST & $9 / 19,5$ & 15,6 & $0,88 / 0,2$ & 0,79 & $0,23 / 0,35$ & 0,42 & \\
\hline
\end{tabular}

RF: resultado funcional; GS: gravedad de los síntomas; DST: desviación estándar.

\section{Tabla III - RESULTADOS FUNCIONALES: ATROFIA TENAR, MOVILIDAD Y FUERZA}

\begin{tabular}{|c|c|c|c|c|c|c|c|c|c|c|c|c|c|c|}
\hline \multirow[b]{2}{*}{ Caso } & \multicolumn{2}{|c|}{ Atrofia Tenar } & \multirow{2}{*}{$\begin{array}{l}\text { Abducción } \\
\text { Palmar } \\
\text { Máxima (cm) }\end{array}$} & \multirow{2}{*}{$\begin{array}{c}\text { Abducción } \\
\text { radial (") }\end{array}$} & \multirow{2}{*}{$\begin{array}{l}\text { Oposición } \\
\text { (Indice de } \\
\text { Kapandjij) }\end{array}$} & \multicolumn{3}{|c|}{ Fuerza PP } & \multicolumn{3}{|c|}{ Fuerza PL } & \multicolumn{3}{|c|}{ Fuerza Puño } \\
\hline & Preax & Postax & & & & $\begin{array}{l}\text { afecto/no } \\
\text { afecto }\end{array}$ & $\begin{array}{c}\% \\
\text { teórico }\end{array}$ & $\begin{array}{c}\% \\
\text { contralateral }\end{array}$ & $\begin{array}{c}\text { afecto/ } \\
\text { no afecto }\end{array}$ & $\begin{array}{l}\% \\
\text { teórico }\end{array}$ & $\begin{array}{c}\% \\
\text { contralateral }\end{array}$ & $\begin{array}{c}\text { afecto/ } \\
\text { no afecto }\end{array}$ & $\begin{array}{l}\% \\
\text { teórico }\end{array}$ & $\begin{array}{c}\% \\
\text { contralateral }\end{array}$ \\
\hline 1 & Mod & Lig & 8 & 50 & 10 & $7,5 / 7$ & 141,5 & 107 & $8 / 8$ & 123 & 100 & $30 / 26$ & 107 & 115 \\
\hline 2 & Grave & Grave & 5 & 60 & 10 & $1 / 4,5$ & 22,7 & 22,2 & $4,7 / 4,5$ & 86,3 & 105,5 & $16 / 16$ & 66,6 & 100 \\
\hline 3 & Grave & Grave & 9 & 40 & 10 & $6 / 7,2$ & 93,7 & 83,3 & $8 / 6,8$ & 100 & 117,6 & $22 / 24$ & 66,6 & 91,66 \\
\hline 4 & Grave & Grave & 6 & 70 & 10 & $5,5 / 6,5$ & 71,4 & 84,6 & $7,5 / 7,5$ & 84,3 & 100 & $19 / 19$ & 48,7 & 100 \\
\hline 5 & Grave & Grave & 7,5 & 50 & 10 & $4 / 6$ & 66,6 & 66,6 & $4,5 / 5,5$ & 62,5 & 81,8 & $10 / 12$ & 32,2 & 83,3 \\
\hline 6 & Grave & Grave & 8,5 & 50 & 10 & $6,5 / 7,5$ & 114 & 86,6 & $6 / 8$ & 92,3 & 75 & 20/22 & 71,4 & 90,90 \\
\hline Media & & & 7,3 & 53,3 & & $5,08 / 6,45$ & 83,5 & 75,1 & $6,45 / 6,71$ & 89,8 & 96,6 & $19,5 / 19,8$ & 64,6 & 96,8 \\
\hline DST & & & & & & $2,3 / 1,09$ & & & $1,6 / 1,4$ & & & $1,6 / 1,4$ & & \\
\hline
\end{tabular}

PL: pulpejo-lateral; PP: pulpejo-pulpejo; DST: desviación estánda

relación a la movilidad del pulgar, todos los pacientes llegaban a la base del $5^{\circ}$ dedo (10 puntos de la escala de Kapandji); la abducción radial media fue de $53,3^{\circ}\left(\right.$ rango $\left.40-70^{\circ}\right)$; y la abducción palmar media de 7,3 cm (rango 6-9 cm). La fuerza media de las pinzas pulpejo-pulpejo, pulpejo-lateral y de puño fueron respectivamente de un $83,5 \%, 89,8 \%$ y $64,6 \%$ de la esperada según tablas de normalidad para edad, género, lado afecto y dominancia ${ }^{18}, 19$; y de un $75,1 \%$, $96,6 \%$ y $96,8 \%$ respecto al lado contralateral (Tabla III).

\section{DISCUSIÓN}

Es poco frecuente encontrar en pacientes con SCC crónico debilidad en la abducción y oposición del pulgar como consecuencia de la denervación del abductor pollicis longus y del opponens pollicis. Incluso en casos de compresión grave la oposición puede estar conservada debido a la inervación mixta mediano-cubital de la musculatura tenar. En la serie más larga publicada en la literatura de liberación del nervio mediano y plastia de Camitz, sólo la encuentran indicada por una inadecuada 
oposición en un $6 \%$ de los pacientes con $\mathrm{SCC}^{1}$. En nuestra serie se realizó en un 2,67\% de los casos. Coincidimos con otros autores en que las plastias son poco frecuentes en los países desarrollados debido al diagnóstico y tratamiento precoces de los síndromes de compresión nerviosa ${ }^{21}$.

En cuanto a los resultados publicados en la literatura, para Foucher et al. ${ }^{1}$ son buenos a largo plazo en el $91 \%$ de los casos con una edad media de 59 años en su serie. Terrono et al. ${ }^{6}$, obtienen un $94 \%$ de resultados satisfactorios, en pacientes con una media de edad de 65 años. En nuestra serie, con una media de edad de casi 64 años, los resultados son satisfactorios en todos los casos. El cambio en la calidad de vida de los pacientes según los cuestionarios DASH y CTS pre y postquirúrgicos valorados con el ES y la SRM denotan una mejoría importante en sus valores tras la intervención. También obtuvimos un buen rango de movilidad del pulgar tanto para la abducción radial y palmar como para la oposición, lo que contribuyó a mejorar la funcionalidad de la mano. Al igual que en nuestra serie otros autores, no encuentran una recuperación significativa de la atrofia tenar ${ }^{1,6}$. Finalmente, los valores de las pinzas pulpejo-pulpejo, pulpejo-lateral y de puño son cercanos e incluso superiores a los teóricos según tablas, excepto para los 2 casos con un SCC contralateral. Para Geere et al. ${ }^{22}$ la medida más sensible para detectar cambios de importancia clínica, o con más respuesta para valorar el resultado de la función motora tras la descompresión del canal carpiano de recuperación de la musculatura tenar, es la pinza pulpejo-pulpejo.

Nuestro estudio presenta una serie de limitaciones: como el discreto tamaño muestral, ya que la indicación de la plastia es cada vez más rara en los países desarrollados. El hecho de poder comparar nuestros resultados con los de otras series tan sólo en términos globales (dado que no hemos encontrado otras series que presenten valores de DASH, CTS, movilidad o fuerza medidos de forma similar a la nuestra). La existencia de factores de co-morbilidad presentes en hasta un $50 \%$ de nuestros casos (un dedo en resorte y De Quervain en un mismo paciente y dos SCC bilaterales). La no disposición de las mediciones pre-tratamiento de la exploración física que obliga a una comparación de las mismas con mediciones realizadas por otros autores en la población general ${ }^{18,19}$. La gran dificultad que implica la gradación de la atrofia tenar según el propio Gelberman ${ }^{9}$, recomendando Geere et al. ${ }^{22}$ la valoración de la atrofia tenar en base a la existencia o no de la misma.

Además los resultados de ES y SRM han ser de valorados con cautela ya que en el $50 \%$ de los casos los valores del DASH y CTS postquirúrgico tienen la mínima puntuación posible, lo que constituye el llamado «efecto suelo» («floor effect»), lo que podría afectar a la validez de estas mediciones como herramienta para valorar los resultados en esta serie.

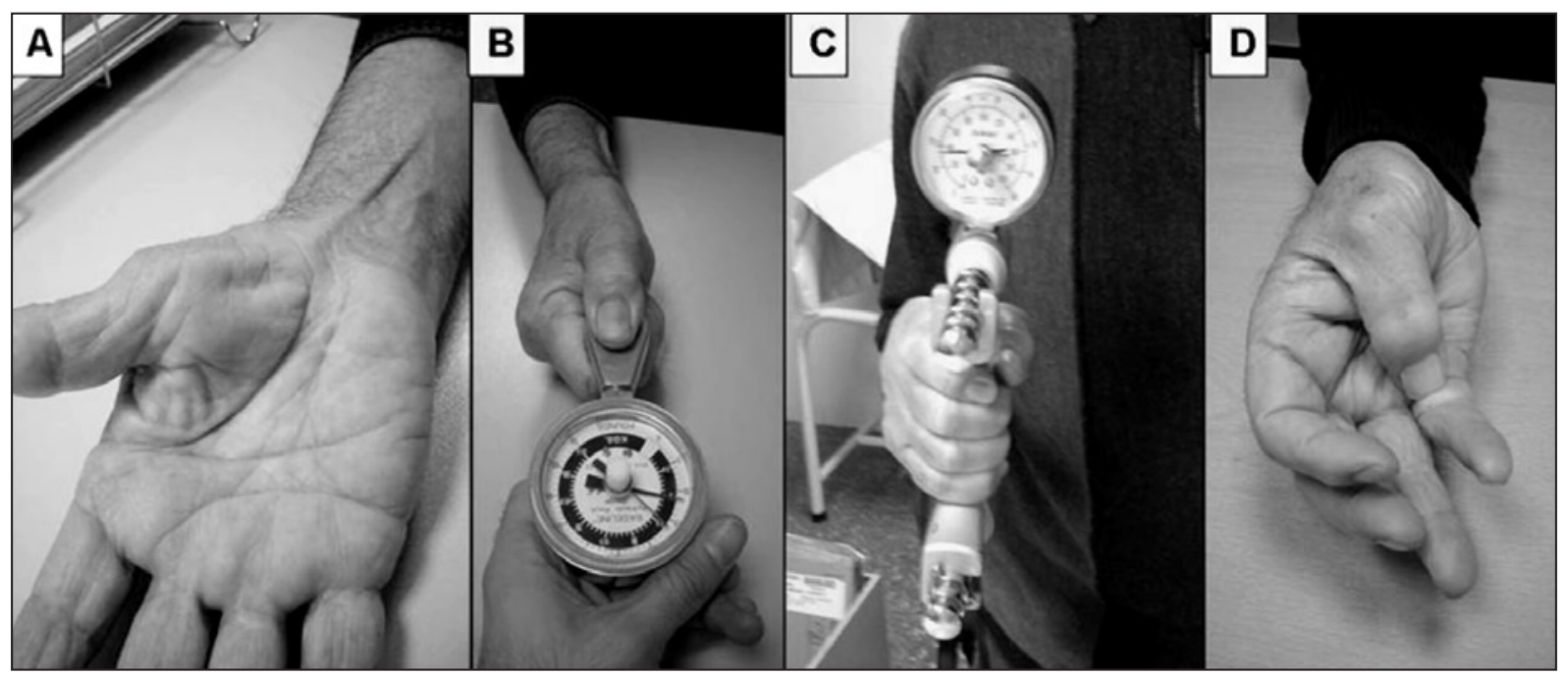

Figura 3. Caso $n^{o}$ 4. A. Persistencia de la atrofia tenar grave. B. Pinza pulpejo lateral de 7,5Kg. C. Fuerza de puño de 19Kg. D. Oposición completa. A pesar de la atrofia, la movilidad del pulgar es buena y su posición en antepulsión favorece la realización de las distintas pinzas. 
De entre las distintas plastias descritas en la literatura, la de Camitz es técnicamente sencilla, puede ser realizada con anestesia regional y no plantea déficit en la zona donante ${ }^{23}$. Aunque esta plastia por sí misma no contribuye directamente a mejorar la fuerza de pinza ni de puño, sí que coloca al pulgar en mejor posición para realizarlas ya que mejora su antepulsión, y hace converger las fuerzas de pronación, flexión y aducción hacia la palma de la mano ${ }^{6,24}$ (Figura 3). Teniendo en cuenta el limitado número de casos de nuestra serie, creemos que aunque no se recupere la atrofia, la oponente-plastia de Camitz es una intervención simple, segura y efectiva que ayuda a mejorar la función de la mano en los casos de SCC crónico de grado grave, especialmente en pacientes mayores.
1. Foucher G, Malizos C, Sammut $\mathrm{D}$, et al. Primary palmaris longus transfer as an opponensplasty in carpal tunnel release. J Hand Surg Br, 1991; 16: 56-60.

2. Nolan WB III, Alkaitis D, Glickel SZ, et al. Results of treatment of severe carpal tunnel. J Hand Surg Am, 1992; 17: 1020-23.

3. Porter P, Venkateswaran B, Stephenson $\mathrm{H}$, et al. The influence of age on outcome after operation for the carpal tunnel syndrome. A prospective study. J Bone Joint Surg Br, 2002; 84: 688-91.

4. Hobby JL, Venkatesh R, Motkur $P$. The effect of age and gender upon symptoms and surgical outcomes in carpal tunnel syndrome. J Hand Surg Br, 2005; 30: 599-604.

5. Braun RM. Palmaris longus tendon transfer for augmentation of the thenar musculature in low median palsy. J Hand Surg Am, 1978; 3: 488-91.

6. Terrono AL, Rose JH, Mulroy J, et al. Camitz palmaris longus abductorplasty for severe thenar atrophy secondary to carpal tunnel syndrome. J Hand Surg Am, 1993; 18: 204-6.

7. Anderson GA, Lee V, Sundararaj GD. Opponensplasty by extensor indicis and flexor digitorum superficialis tendon transfer. J Hand Surg Br, 1992; 17: 611-4.

8. Camitz H. Uber die Bhenadlung der Oppositionslahmung. Acta Chir Scand, 1929; 65: 77- 81.

9. Gelberman RH, Pfeffer GB, Galbraith RT, et al. Results of treatment of severe carpal tun-

\section{BIBLIOGRAFÍA}

nel syndrome without internal neurolysis of the median nerve. $J$ Bone Joint Surg Am, 1987; 69: 896-903.

10. Graham B, Regehr G, Naglie $\mathrm{G}$, et al. Development and validation of diagnostic criteria for carpal tunnel syndrome. J Hand Surg Am, 2006; 31: 919, e1-7.

11. Shin AY, Dao KD. Tendon transfers for thumb opposition. Atlas Hand Clin, 2002; 7: 1: 1-17.

12. Rosales RS, Delgado EB, Diez de la Lastra-Bosch I. Evaluation of the Spanish version of the DASH and carpal tunnel syndrome health-related quality-of-life instruments: crosscultural adaptation process and reliability. J Hand Surg Am, 2002; 27: 334-46.

13. De Smet, L. Responsiveness of the DASH score in surgical treated basal joint arthritis of the thumb: preliminary results. Clin Rheumatol, 2004; 23 : 223-4.

14. Rosales RS, Diez de la Lastra I, McCabe S, et al. The relative responsiveness and construct validity of the Spanish version of the DASH instrument for outcomes assessment in open carpal tunnel release. J Hand Surg Eur, 2009; 34: 72-5.

15. Kazis L, Anderson JJ, Meenan RF. Effect sizes for interpreting changes in health status. Med Care, 1989; 27: S178-89.

16. Van Wetter, P. Le champ d’action du pouce et ses limites. Ann Chir, 1974; 28: 851-3.

17. Kapandji A. Cotation c1inique de l'opposition et de la contre- oppositiondu pouce. Ann Chir Main, 1986 ; 5: 67-73.

18. Mathiowetz V, Kashman N, VoIland $G$, et al. Grip and Pinch strength: normative data for adults. Arch Phys Med Reh, 1985; 66: 69-72.

19. Torres-Coscoyuela M, González-del Pino J, Yánez-Calvo J, et al. Estudio dinamométrico de la mano y el pulgar. Rev Ortop Traumatol, 1999; 43: 321-6.

20. Liang $\mathrm{MH}$, Fossel $\mathrm{AH}$, Larson MG. Comparisons of five status instruments for orthopaedic evaluation. Med Care, 1990; 28: 632-42.

21. Botelheiro JC, Silverio S, Viana G. Atrofia ténar em sindromas de túnel cárpico graves-21 casos operados. Rev Iberamer Cir Mano, 2007; 35: 22-6.

22. Geere J, Chester R, Kale S, et al. Power grip, pinch grip, manual muscle testing or thenar atrophy-which should be assesed as a motor outcome after carpal tunnel decompression? A systematic review. BMC Musculoskelet Disord, 2007; 8: 114.

23. Ting J, Weiland AJ. Role of ancillary procederes in surgical management of carpal tunnel syndrome: epineurotomy, internal neurolysis, tenosynovectomy, and tendon transfers. Hand Clin, 2002, 18: 315: 23.

24. Brand PW, Hollister AM: Operations to restore muscle balance to the hand. En: Clinical Mechanics of the Hand. $3^{a} \mathrm{Ed}$. St Louis, Baltimore: CV Mosby, 1999: 274-321. 